\title{
A rare case of a patient with a high grade neuroendocrine tumor developing neutropenic sepsis after receiving PRRT combined with Capecitabine or Temozolomide: A case report
}

\author{
BURCIN ÖZDIRIK ${ }^{1}$, HOLGER AMTHAUER ${ }^{2}$, IMKE SCHATKA ${ }^{2}$, PETER E. GORETZKI ${ }^{3}$, MARTINA T. MOGL ${ }^{3}$, \\ ULI FEHRENBACH $^{4}$, FRANK TACKE ${ }^{1}$, HENNING JANN ${ }^{1 *}$ and CHRISTOPH RODERBURG ${ }^{1 *}$ \\ Departments of ${ }^{1}$ Hepatology and Gastroenterology, ${ }^{2}$ Nuclear Medicine, ${ }^{3}$ Surgery and \\ ${ }^{4}$ Diagnostic and Interventional Radiology, Charité-Universitätsmedizin Berlin, corporate member of Freie \\ Universität Berlin, Humboldt-Universität zu Berlin, and Berlin Institute of Health, Berlin 13353, Germany
}

Received January 19, 2020; Accepted August 28, 2020

DOI: $10.3892 / \mathrm{mco} .2020 .2182$

\begin{abstract}
Neuroendocrine tumors (NET) are rare and demonstrate variable clinical behavior depending on the degree of tumor differentiation. Patients with poorly differentiated tumors (NET G3) have a poor prognosis. Systemic treatment with cytotoxic chemotherapy is considered to be the treatment of choice. In patients that are refractory or intolerant to first-line therapy, experts recommend peptide receptor radionuclide therapy (PRRT) in tumors that express somatostatin receptors. Recently, combinations of PRRT and chemotherapy were tested in patients with NET. Available data have reported promising tumor control rates and an excellent toxicity profile in cases where PRRT had been administered with capecitabine/temozolomide, even when administered as salvage therapy. The current study reported an exceptional case of advanced NET G3 with severe toxicity upon receiving PRRT in combination with capecitabine/temozolomide as third line therapy. The patient developed a life-threatening neutropenic fever, fungal pneumonia and necrotizing mastitis
\end{abstract}

Correspondence to: Professor Christoph Roderburg, Department of Hepatology and Gastroenterology, Charité-Universitätsmedizin Berlin, corporate member of Freie Universität Berlin, Humboldt-Universität zu Berlin, and Berlin Institute of Health, Augustenburger Platz 1, Berlin 13353, Germany

E-mail: christoph.roderburg@charite.de

*Contributed equally

Abbreviations: CUP, cancer of unknown primary; ECOG, Eastern Cooperative Oncology Group; GBq, gigabecquerel; G, grade; $\mathrm{MBq}$, megaBq; mGy, miligrays; NEN, neuroendocrine neoplasia; NET, neuroendocrine tumors; PRRT, peptide receptor radionuclide therapy; PET, positron emission tomography; PFS, progression-free survival; SSA, somatostatin analogue; SSTR, somatostatin receptors

Key words: peptide receptor radionuclide therapy, capecitabine, temozolomide, neuroendocrine poorly differenciated tumors, hematologic toxicity, response
23 days after the first cycle of therapy was administered. However, the treatment led to a significant reduction in tumor size. A total of 5 months after treatment initiation, the patient was alive and in excellent clinical condition with sustained tumor response. In summary, the current study presented a rare case of high grade NET exhibiting an almost complete response to PRRT in combination capecitabine/temozolomide, despite facing unexpected severe toxicity.

\section{Introduction}

Neuroendocrine neoplasia (NEN) are a rare and heterogeneous group of tumors. According to the World Health Organization (WHO) classification, NEN are stratified into low/moderate-[grade (G) 1/2] or high-grade (G3) neuroendocrine tumors (NET) and neuroendocrine carcinoma (NEC) (1). Well-differentiated NET (G1 and G2) are characterized by a low proliferative index, retain the expression of somatostatin receptors (SSTR) and are associated with a good prognosis compared with that in other malignancies. By contrast, G3 NET feature a high Ki-67 proliferation index of $>20 \%$ and are associated with a poor prognosis.

The systemic treatment of patients with G3 NET has been under investigated. To date, no data from prospective clinical trials are available, and current recommendations for the treatment of G3 NET primarily relies on retrospective analyses and case series. Overall, G3 NET show low objective response rates to platinum-based therapies, when compared with that in NECs (2). Therefore, alternative, less toxic chemotherapy regimens, such as capecitabine/temozolomide are recommended (3). Data on second- or third-line therapy in the treatment of G3 NET are even rarer, and current recommendations are primarily based on expert opinions rather than on systematic clinical studies. In the case of SSR-positive tumors, peptide receptor radionuclide therapy (PRRT) has been recommended by several expert research groups. PRRT is a tumor-targeted systemic radiotherapy that enables the specific delivery of radionuclides directly into tumor cells inducing tumor cell death. The high-level expression of SSR on the tumor cell surface in NEN provides the rational for a therapy 
with radioisotope-labeled somatostatin analogs (4). While PRRT has emerged as a highly effective and well-tolerated treatment in SSR-positive, well-differentiated NET (5-7), few data exist on patients with high-grade NET. Zhang et al (8) reported a median progression-free survival (PFS) time of 9.6 months and a median overall survival (OS) time of 19.9 months in 69 patients with G3 NET treated with PRRT. Notably, in these patients PRRT was well-tolerated without any decline in renal function, hepatotoxicity or grade 3/4 hematotoxicity. Combinations of PRRT with systemic chemotherapy (e.g. capecetabine with and without temzolomide) might be associated with both additive and synergistic effects, since chemotherapeutic agents might serve as a radiosensitizer, as well as targeting cells non-responsive to PRRT (9). However, at present, there are only a few case reports and small number of case series, which have reported the outcome of patients treated with a combination of PRRT and chemotherapy. In the present case study, a patient with disease progression following 4 cycles of PRRT, who was subsequently treated with a combination of PRRT and capecitabine/temozolomide at our institution has been described.

\section{Case report}

The case of a 58-year-old female patient who was diagnosed with a G3 NET of unknown primary location and synchronous liver metastases in October 2017 (Table I) has been described. The proliferation according to $\mathrm{Ki}-67$ was high (20\%). Multi-slice computed tomography (CT) and DOTATOC-positron emission tomography (PET)/CT revealed multiple SSR-positive liver metastases; however, it did not provide any evidence of a primary tumor. Immunohistochemical analysis of a biopsy obtained from a liver metastasis showed strong expression of synaptophysin and a slightly weaker expression of chromogranin. Staining for serotonin, CDX2 and TTF1 were negative and membranous PD-L1 expression was found in $<1 \%$ of tumor cells.

Treatment with lanreotide Autogel (120 mg) was administered every 28 days. In addition, the patient underwent 4 cycles of PRRT with 7.4 gigabecquerel (GBq) ${ }^{177} \mathrm{LU}$ LU-DOTATOC over a period of 6 months (last dose June 2018). Notably, this treatment resulted in a partial remission lasting until December 2018 (Fig. 1A-C). At this time point a follow-up DOTATOC-PET/CT scan revealed disease progression in the liver (only in the right lobe, with stable disease on the left-hand side). No other distant metastases was evident. Based on the short duration of tumor control, another systemic treatment was not administered; however, the patient was admitted to undergo hemihepatectomy to resect the progressive lesions. Histopathological analysis of the resected tumor confirmed the diagnosis of NET with $\mathrm{Ki}-67>20 \%$, leading to the diagnosis of G3 NET (Fig. 2A-C). However, a DOTATOC-PET/CT scan performed four months following surgery showed further hepatic and lymphatic progression with an increase in tumor size of $>20 \%$ according to the Response Evaluation Criteria in Solid Tumors (RECIST). Considering the initial partial response to PRRT and the systemic progression at that time point, systemic therapy was not administered but simultaneously continuation of PRRT sessions. Capecitabine/temzolomide was chosen as the chemotherapeutic agent due to the high response rates observed in patients with NET $(10,11)$ and since it represents the most common therapy regime used in studies investigating PRRT in combination with chemotherapy (12-14). At the time point of treatment initiation, the patient was in good general condition [Eastern Cooperative Oncology Group (ECOG) 0] and had recovered from the side effects of previous therapies. Nevertheless, the therapy was administered at a reduced dose, since (reversible) anemia and a lower platelet and leucocyte count had occurred, as some of the side effects from the initial 4 cycles of PRRT (Fig. 3A and B).

As timing between chemotherapy and PRRT has been found to have an impact on outcome parameters in animal studies, wean already established protocol was used $(15,16)$. Of note, this particular protocol was selected, as it has been reported that therapy was only accompanied by modest reversible myelosuppression, which was not greater than that in conventional PRRT therapies. Therefore, the combination of PRRT plus capecitabine/temozolomide was administered according to the protocol recently published by Strosberg et al (10) using $750 \mathrm{mg} / \mathrm{m}^{2}$ capecitabine (which was reduced to $538 \mathrm{mg} / \mathrm{m}^{2}$ ) and temzolomide $200 \mathrm{mg} / \mathrm{m}^{2}$ (which was reduced to $150 \mathrm{mg} / \mathrm{m}^{2}$ ). Chemotherapy with oral capecitabine started five days prior to PRRT. In particular, 7.0 GBq ${ }^{177}$-LU-DOTATOC was administered intravenously, followed by oral temozolamide in the last five days of the 14-day period of the capecitabine cycle. Dosimetric calculations revealed that the radiation absorbed doses were 1.09 milligrays $(\mathrm{mGy}) / \mathrm{megaBq}(\mathrm{MBq})$ for the kidneys, $0.288 \mathrm{mGy} / \mathrm{MBq}$ for the liver, $0.41 \mathrm{mGy} / \mathrm{MBq}$ for the spleen and $0.03 \mathrm{mGy} / \mathrm{MBq}$ for bone marrow, while hepatic metastases demonstrated a higher uptake of $4.56 \mathrm{mGy} / \mathrm{MBq}$, which was in line with previously published results from patients receiving combinations of chemotherapy and PRRT (17).

The treatment was initially well-tolerated without any side effects. However, 23 days after PRRT, the patient was hospitalized due to recurrent episodes of fever, dyspnea, as well as pain, redness and swelling in the right mamma. Laboratory testing revealed pancytopenia and slightly elevated inflammatory markers, while an ultrasound of the mamma showed distinct edema with induration of the tissue without evidence of an abscess formation. Chest CT revealed a mass-like pulmonary infiltrate in the right upper lobe with surrounding ground glass opacity, suggesting fungal pneumonia (Fig. 4A and B).

Blood, sputum and swab cultures did not identify any pathogens. For further evaluation of the pancytopenia, a bone marrow puncture was performed, which revealed toxic bone marrow damage (Fig. 5). As a result of the clinical investigations, neutropenic fever, a right-sided necrotizing mastitis and fungal pneumonia, as clinical complications of toxic bone marrow aplasia (most likely due to hematoxicity of PRRT and chemotherapy) was diagnosed. The patient, treated in an external rural hospital at that time, was then sent to an Oncology unit in a tertiary University hospital. An empirical combination therapy with piperacillin/tazobactam, vancomycin, aciclovir and Caspofungin was initiated and was later changed to a combination of vancomycin, ciprofloxacin and voriconazole. Furthermore, stimulation therapy with granulocyte-colony stimulating factor was performed, along with the administration of several red blood cell and platelet transfusions. After 11 days, the blood cells started to regenerate (Fig. 3A and 3B). Follow-up imaging two weeks 
Table I. Course of disease.

\begin{tabular}{lll}
\hline Year Month $\quad$ Therapy & Staging
\end{tabular}

2017 October

November

SSA therapy (Somatuline $120 \mathrm{mg}$ ) every 28 days

December

First cycle PRRT 7,4 GBq

${ }^{177}$ LU-DOTATOC

2018 February

April

June

July

Fourth cycle PRRT 7,7 GBq

${ }^{177}$ LU-DOTATOC

Continuation of SSA therapy

September

December

2019 January

Right hemihepatectomy

May

June

Fifth PRRT 6,941 GBq ${ }^{177} \mathrm{Lu}$-DOTATOC in combination with capecitabine $\left(540 \mathrm{mg} / \mathrm{m}^{2}\right)$ and temzolomide $\left(150 \mathrm{mg} / \mathrm{m}^{2}\right)$

July Hospitalization due to clinical complications (neutropenic fever, transfusion obligatory pancytopenia, right sided necrotizing mastitis, fungal pneumonia) after PRRT in combination with capecitabine/temzolomide August Continuation of SSA therapy

October
G3 NET CUP with synchronous hepatic metastases: First biopsy of a hepatic metastasis, Ki $6720 \%$, Synaptophysin+++, CGA++, SSTR-2A+++ Staging CT and DOTATOC-PET: SSR-positive multiple hepatic metastases primarily in the right liver lobe. No evidence of primary tumor

Second biopsy of a hepatic metastasis: Ki67 35-40\%, Synaptophysin+++, CGA++, SSTR-2A+++, ISLET1-positive, TTF1- and CDX2-negative

Staging CT: Hepatic progressive disease

Staging CT and DOTATOC-PET: Hepatic progressive disease

Staging CT: Partial remission with hepatic tumor size reduction

Staging CT and DOTATOC-PET: Partial

further hepatic tumor size reduction

Staging CT and DOTATOC-PET: Hepatic progressive disease (progress of right lobe liver metastasis).

No pathological lymph node enlargement

Histopathology of liver specimen: Ki $67>20 \%$, synaptophysin +++, CGA++, MLH1+, MSH2+, MSH6+, PMS2+serotonin, CDDX2 and TTF1 negative. PD-L1+ Staging CT and DOTATOC-PET: Hepatic and lymphatic progressive disease ( $>20 \%$ according to RECIST)

Staging CT: Partial remission (55\% according to RECIST).

No pathological lymph node enlargement. No primary tumor detectable

MR: Partial remission (68\% according to RECIST)

PRRT, peptide receptor radionuclide therapy; SSA, somatostatin analogue therapy; CT, computer tomography; MR, magnetic resonance; PET, positron emission tomography; RECIST, Response Evaluation Criteria in Solid Tumors.

later revealed a clear regression of the infiltrations in the right upper lobe. The inflammatory markers decreased, along with an improvement in the healing process of the wound tissue of the mamma (Fig. 6).

Despite the critical clinical condition caused by combination PRRT, CT staging conducted one month following PPRT plus capecitabine/temozolomide showed a liver tumor mass reduction of at least $55 \%$ according to RECIST, without any signs of pathological lymph node enlargement. Subsequent magnetic resonance imaging four months later revealed a further tumor reduction of at least $68 \%$ according to RECIST (Fig. 1D and E).

In consideration of the severe bone marrow damage and the critical condition of the patient, the combination of PRRT plus capecitabine/temozolomide was discontinued and somatostatin analogue (SSA)-therapy was restarted again. The 
A

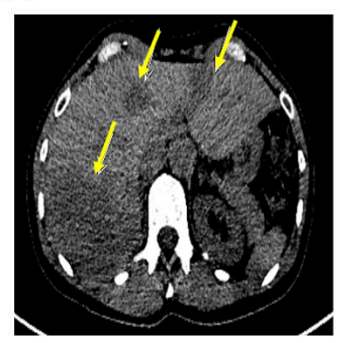

B

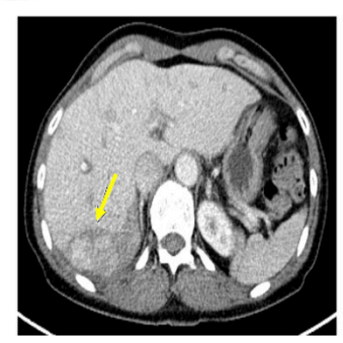

C

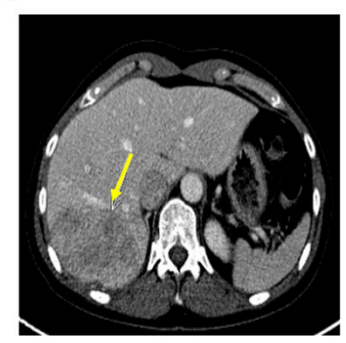

D

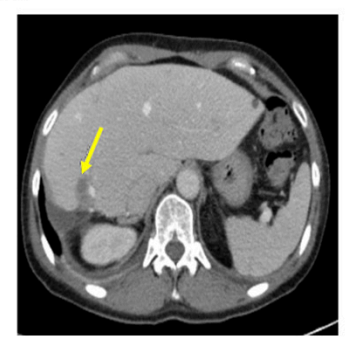

E

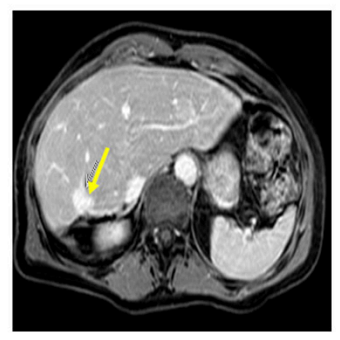

Figure 1. Hepatic metastases in axial abdominal CT and MRI scans during the course of treatment in a 58-year-old female patient with neuroendocrine CUP and synchronous hepatic metastases. Tumor lesions are indicated by yellow arrows. (A) Axial non-contrast-enhanced CT scan demonstrates multiple hepatic metastases at initial diagnosis (November 2017). (B) Axial-contrast enhanced (venous phase) CT scan displays a reduction in the size of hepatic metastases after 4 cycles of PRRT (July 2018). (C) Axial-contrast enhanced (venous phase) CT scan demonstrates progression in the size of hepatic metastases primarily in the right liver lobe (December 2018). (D) Axial contrast-enhanced (venous phase) CT scan demonstrates tumor reduction of at least 55\% (according to RECIST v11.1) 1 month after PRRT in combination with capecitabine/temzolomide (July 2019). (E) Axial contrast-enhanced (venous phase) MRI depicts a hepatic tumor reduction of at least 68\% (according to RECIST v11.1) after one cycle of PRRT in combination with capecitabine/temozolomide (October 2019). CUP, cancer of unknown primary; PRRT, peptide receptor radionuclide therapy; RECIST, Response Evaluation Criteria in Solid Tumors.

A

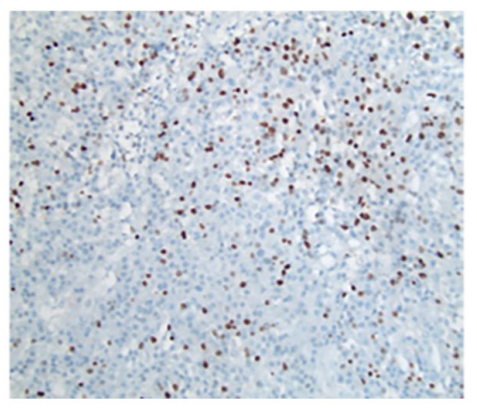

B

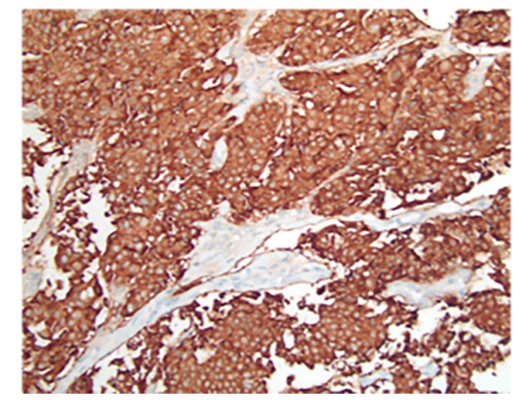

C

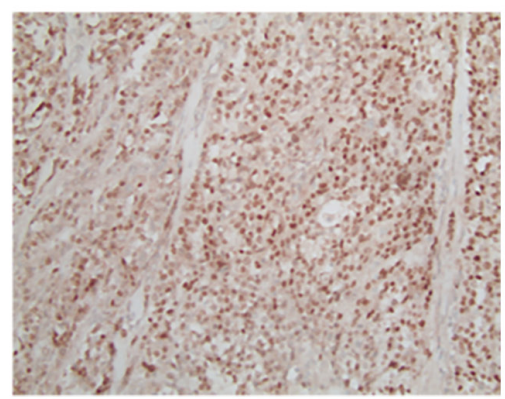

Figure 2. Immunohistochemical expression of cells from hepatic metastasis (hemihepatectomy resection from January 2019). Pathological analysis and immunohistochemical expression of cells from hepatic metastasis obtained following hemihepatectomy resection. (A) Ki-67 expression was $>20 \%$. (B) Synaptophysin expression of tumor cells. (C) Chromogranin expression of the tumor cells (all, magnification x20).

A

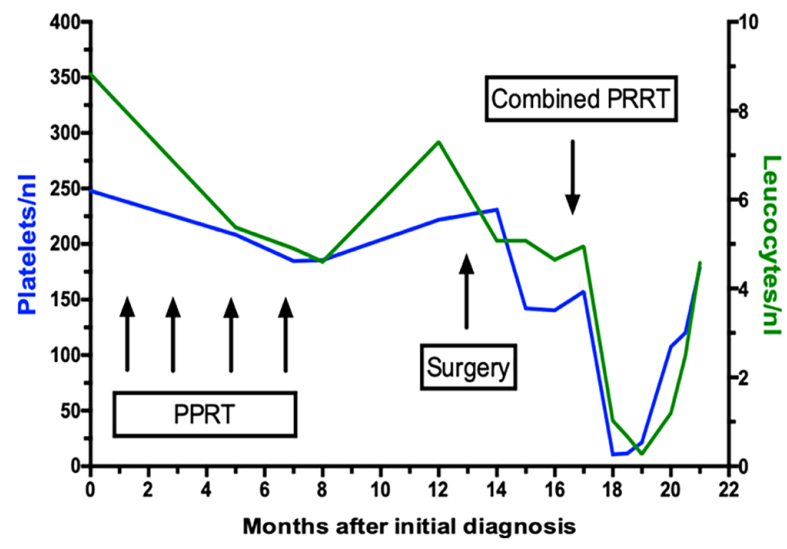

B

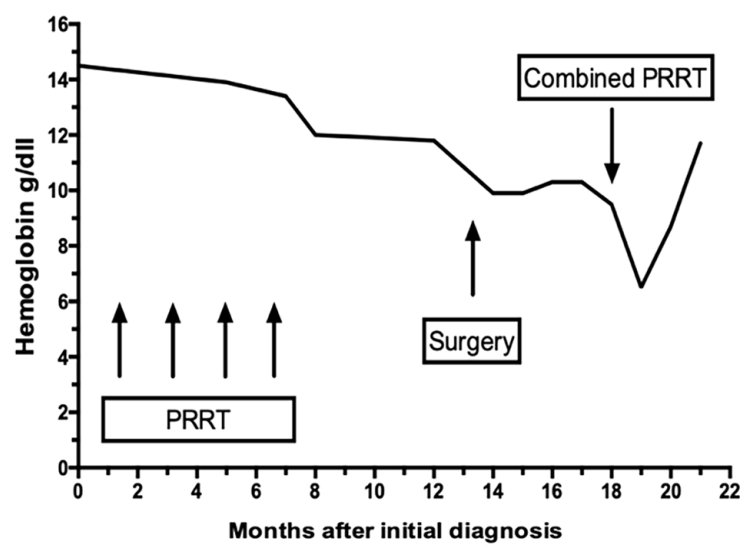

Figure 3. Platelet and leucocyte count during the course of treatment. (A) Platelet (blue curve) and leukocyte count (green curve) during the course of treatment. The graph illustrates a large decrease in leucocyte and platelet count 23 days after PRRT in combination with capecitabin/temzolomid. The results also exhibit a decline in platelet and leucocyte count after the administration of four cycles of PRRT, which rapidly increased subsequently, and stabilized during partial remission, with a decrease as the disease progressed. (B) Hemoglobin levels during the course of treatment. The graph illustrates a progressive decrease in hemoglobin levels after initial diagnosis resulting in chronic anemia. Despite a decrease in hemoglobin levels after four cycles of PRRT, the anemia may have been cancer-related, showing a decline with progressive disease. Subsequent to granulocyte-colony stimulating factor stimulation and the administration of erythrocyte concentrates during hospitalization in July 2019, hemoglobin levels normalized. PRRT, peptide receptor radionuclide therapy.

blood count stabilized and remained normal. The following staging investigations in October 2019 revealed further sustained tumor response. To date, the patient is alive and fully recovered from the therapy-related side effects. 


\section{A}

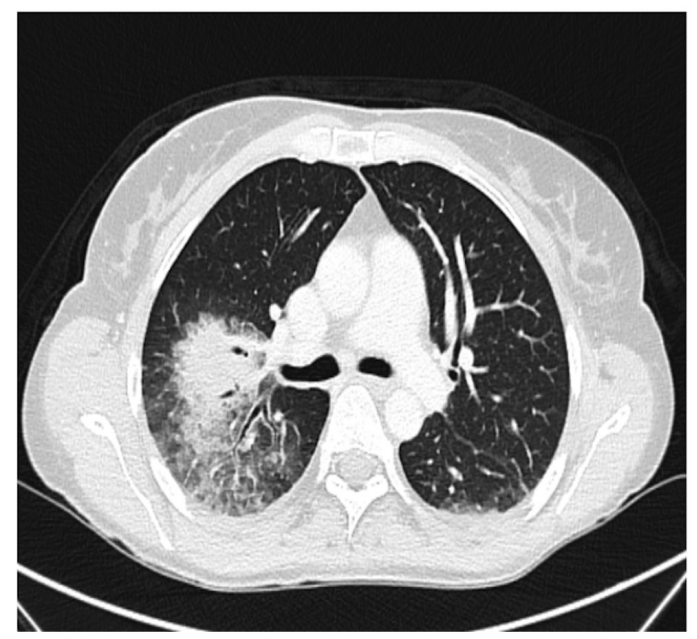

B

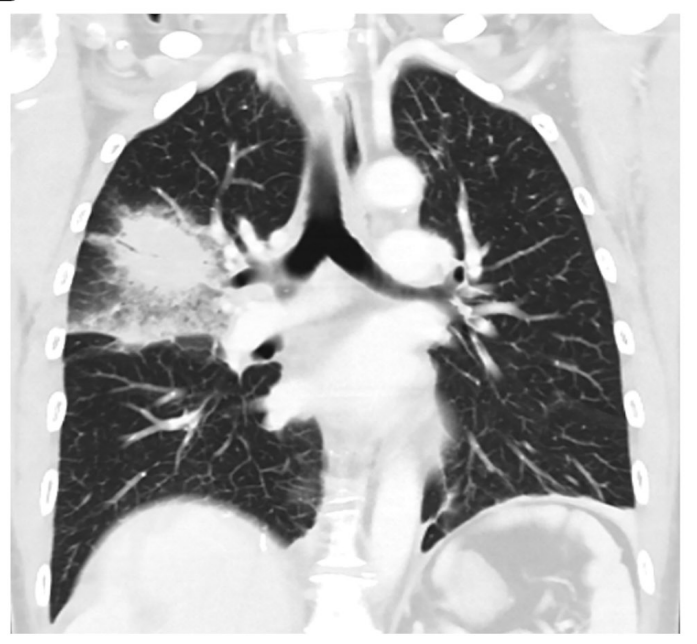

Figure 4. Axial contrast-enhanced CT scan of the chest, suggesting fungal pneumonia. (A) Axial- and (B) coronal contrast-enhanced CT scan of the chest demonstrated a mass-like pulmonary infiltrate in the right upper lobe with surrounding ground class opacity, suggesting fungal pneumonia.

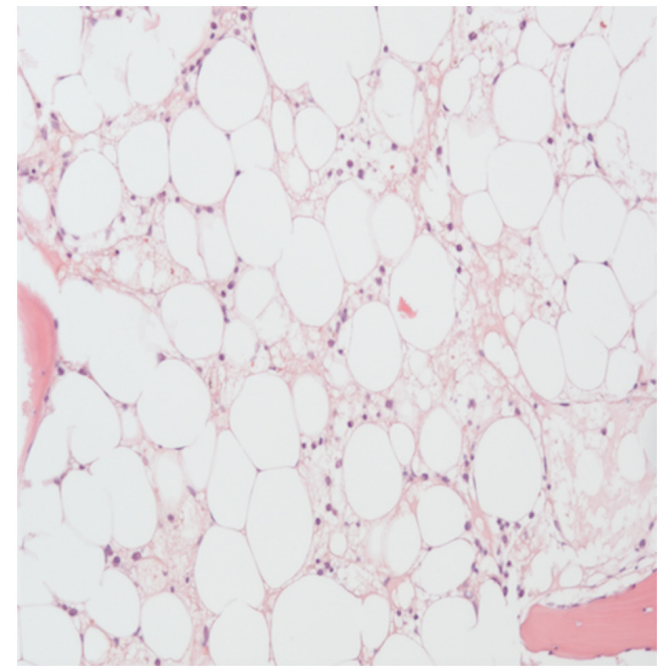

Figure 5. Bone marrow aspirate stained with H\&E showing severe bone marrow hypoplasia. For further evaluation of the unexplained pancytopenia, a bone marrow puncture was performed. Results suggested that toxic bone marrow damage most likely due to hematoxicity of PRRT and chemotherapy.

\section{Discussion}

The present case report described the case of a patient diagnosed with a G3 NET of unknown primary origin accompanied by synchronous liver metastases. The patient received a combination of PRRT and capecitabine/temozolomide chemotherapy, as part of a multi-modal treatment concept at our institution. After receiving only one cycle of therapy, the patient exhibited severe bone marrow toxicity, as well as neutropenic fever and critical infectious complications (necrotizing mastitis and fungal pneumonia); however, demonstrated an effective tumor response. The patient in the present case report provides several notable aspects: First, the combination of chemotherapy and PRRT was associated with an effective tumor response, leading to a sustained tumor control $>5$ months after only one cycle; second, this response was achieved in a patient with high-grade NET, representing a cohort of patients with limited treatment options; third, the toxicity of the treatment exceeded the toxicity reported in the current literature by far, highlighting the requirement for careful patient selection and close monitoring of patients receiving PRRT in combination with chemotherapy.

Until now, different experimental approaches and strategies have been investigated to optimize the effectiveness of PRRT and to minimize potential side effects (18). Research groups, such as Claringbold et al (12-14) have tried to combine PRRT with chemotherapy (capecitabine with and without temzolomidect) in cases of patients with advanced low-grade GNETs, in which either of the two treatment options alone failed $(12-14,16)$. With the intention to use chemotherapy, as a radiosensitizing agent to enhance the efficacy of PRRT, effective tumor control rates were achieved, with disease control in up to $55 \%$ of the patients $(13,19)$. A study, investigating pNET in particular, revealed an overall response rate of $80 \%$, including complete remission in $13 \%$ and partial response in $70 \%$ of the cases (14). Accordingly, the effective tumor response of at least $68 \%$ tumor reduction was in line with previous studies.

Both combined PRRT and PRRT alone have been presented as procedures leading to an increase in long-term survival with a low complication rate (20-28). The patient in the present case study received the combination of ${ }^{177} \mathrm{Lu}$-octreotate and capecitabinec and temozolomide, which was considered feasible and safe, regarding the acute and subacute side effects (12-14). According to previous studies, acute side effects are typically mild and self-limiting (most commonly nausea), whereas long-term side effects include loss of renal function, myelodysplastic syndrome and acute leukemia. However, hematological toxicity was the most significant potential adverse event following PRRT, caused by irradiation of the bone marrow and primarily presenting as reversible, limited grade cytopenia. Current research studies suggest that WHO grade 3 or 4 toxicity could only occur in up to $15 \%$ of patients. According to Kesavan et al (16) this number was not significantly increased in patients receiving PRRT in combination with radiosensitizing chemotherapy, which has the potential to enhance the efficiency of the therapy. Research by Kesavan et al (16) retrospectively analyzed long-term outcomes 
A

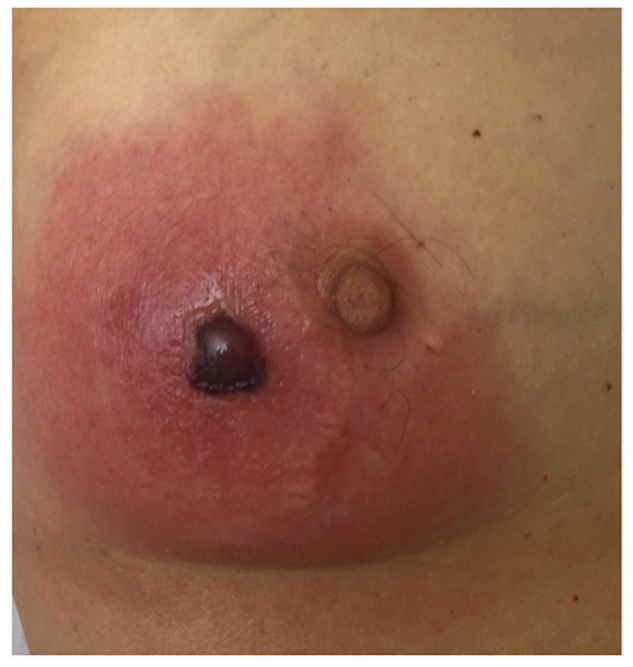

B

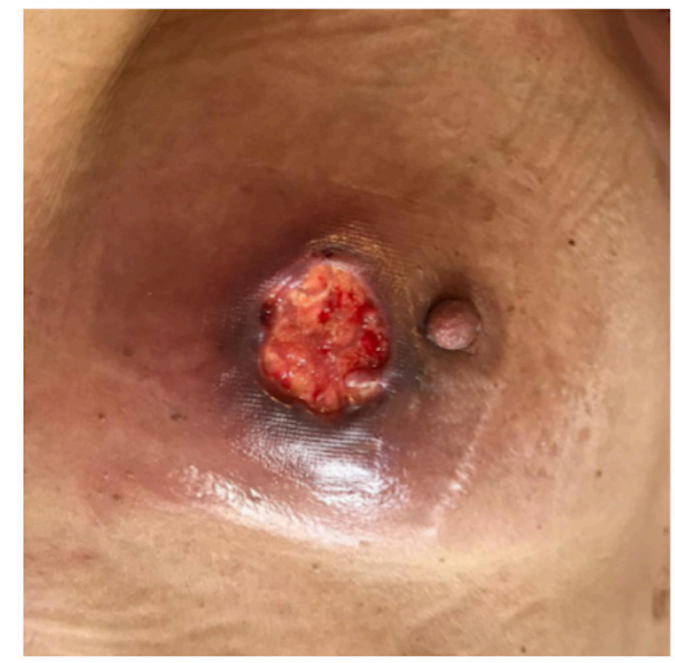

Figure 6. Necrotizing mastitis (with cutis and subcutis involvement) of the right mamma prior to and during treatment. (A) Mastitis before treatment initiation, showing erythema, edema and necrosis. Previous ultrasound imaging of the patient's mamma showed distinct edema with induration of the tissue without evidence of an abscess formation. (B) Mastitis of the right mamma during the healing process after treatment initiation. As a result of therapy, edema and erythema regressed, while necrosis could still be detected.

of the two cohorts from their ${ }^{177} \mathrm{Lu}$-octreotate and chemotherapy study (37 patients treated with capecitabine/temzolomide and 28 patients treated with ${ }^{177} \mathrm{Lu}$-octreotate and capecitabine). In both cohorts, only modest reversible myelosuppression was observed. In patients treated with capecitabine/temzolomide, long-term follow-up revealed significant thrombocytopenia in $2.7 \%(n=1)$, neutropenia in $2.7 \%(n=1)$ and anemia in $10.8 \%(n=4)$, while no short-term hematological toxicity grade $3 / 4(n=0)$ was reported. In patients receiving ${ }^{177} \mathrm{Lu}$-octreotate and capecitabine, long-term hematotoxicity, such as anemia and thrombytopenia was only detected in $3.5 \%$ of the cases $(n=1)$. However, an exact measure of the adverse events due to PRRT plus chemotherapy can be challenging, which is why the procedure is still considered investigational (29).

The patient in the present case report developed severe bone marrow toxicity, along with critical infectious complications (necrotizing mastitis and fungal pneumonia) after only one session of PRRT in combination with capecitabine/temzolomidect at a reduced dose. Despite the fact that only one cycle of combined PRRT, at a reduced dose was administered, severe bone marrow damage was observed, leading to myelotoxic cytopenia most likely caused by prior therapy with PRRT, which was not seen in association with previous SSA therapy $(30,31)$. Fig. 3A and B revealed the myelotoxic damage after two PRRT sessions causing a lower platelet and leucocyte count,counts as well as persistent anemia after several months. However, an increased radiation uptake can be excluded, as dosimetric calculations revealed the radiation absorption doses, which were in line with previously published results from patients receiving combinations of chemotherapy and PRRT (17). Therefore, it was concluded Therefore, we can conclude that the patient in the current study was already predisposed to develop pancytopenia during PRRT in combination with capecitabine/temzolomide. Pretreatment with radiation-based therapy or alkylating agents has also been considered a significant factor to predict myelotoxicity, as research by Kesavan et al (16) showed a significant differencesignificance between increased risk of short- and long-term toxicity and the presence and number of previous treatments. Thus, a reduced dose of capecitabine/temzolomide was administered to the patient in the present case report.

As aforementioned, there are several approaches to prevent adverse effects of PRRT, such as using amino acid infusion or gelofusine and optimization of antiemetic regimens (32-35). Furthermore, it has been suggested that early therapy with PRRT-containing regimens could not only improve the outcome, but also reduce myelotoxicity (36). However, early treatment with PRRT was not successful in preventing severe bone marrow damage in the patient in the present case report, suggesting the requirement for additional approaches to prevent myelotoxicity. In this regard, establishment of specific algorithms incorporating predictors for myelotoxicity are highly desirable to select optimal treatment strategies, with respect to dosage and the number of cycles for each individual patient.

Another primary finding of the present case report was that the tumor reduced in size by at least $55 \%$ after only one month, followed by a further reduction of up to $68 \%$ (Fig. 1D). This supports several previous studies, which consider PRRT in combination with radiosensitizing chemotherapy an effective therapeutic option in this challenging disease (12-14). The rapid response seen in the patient in the current case report indicates the requirement for close clinical and radiological monitoring in patients treated with such regimens, to adjust the therapeutic strategy according to its efficacy and toxicity. Long-term follow-up would be a requirement to investigate sustainability of the tumor response after one cycle, as well as the occurrence of long-term adverse effects.

However, the present case report has some limitations, as only one patient with radiosensitizing chemotherapy in combination with PRRT was treated at our institute, which makes further conclusions difficult. Furthermore, the primary tumor in the patient is still unknown. However, there is a high incidence of, CUP (10-15\%) in patients with NET (37-40) and no correlation between an improveda therapy response 
and/or higher toxicity with respect to tumor origin after PRRT combinationcombined with chemotherapy has been analyzed or reported yet $(12-14,19)$.

Despite the high tumor reduction rate and several successful approaches to reduce the side effect profile in the field of radio sensitizing chemotherapy in combination with PRRT, the serious problem of myelotoxicity could not be addressed. Clinical trials on this type of therapy are rare, but are urgently required to further investigate the toxicity, as well as to develop preventive measures and predictors of response and long-term survival in patients receiving a combination of PRRT and systemic chemotherapy.

\section{Acknowledgements}

Not applicable.

\section{Funding}

No funding was received.

\section{Availability of data and materials}

The datasets used and/or analyzed during the present study are available from the corresponding author on reasonable request.

\section{Authors' contributions}

BÖ, HA, IS, PEG, MTM, UF, FT, HJ and CR were involved treated the patient. BÖ, HJ and CR wrote the manuscript. All authors read and approved the final manuscript.

\section{Ethics approval and consent to participate}

Not applicable.

\section{Patient consent for publication}

The patient provided written informed consent for the publication of patient data and images according to the Declaration of Helsinki.

\section{Competing interests}

The authors declare that they have no competing interests.

\section{References}

1. Bosman FT, Carneiro F, Hruban RH and Theise ND Classification of tumours of the digestive system. 4th edition ed. Geneva, Switzerland: WHO Press; 2010.

2. Vélayoudom-Céphise FL, Duvillard P, Foucan L, Hadoux J, Chougnet CN, Leboulleux S, Malka D, Guigay J, Goere D, Debaere T, et al: Are G3 ENETS neuroendocrine neoplasms heterogeneous? Endocr Relat Cancer 20: 649-657, 2013.

3. Heetfeld M, Chougnet CN, Olsen IH, Rinke A, Borbath I, Crespo G, Barriuso J, Pavel M, O'Toole D, Walter T; Other Knowledge Network members: Characteristics and treatment of patients with G3 gastroenteropancreatic neuroendocrine neoplasms. Endocr Relat Cancer 22: 657-664, 2015.

4. Fani M, Maecke HR and Okarvi SM: Radiolabeled peptides: Valuable tools for the detection and treatment of cancer. Theranostics 2: 481-501, 2012.
5. Kwekkeboom DJ, de Herder WW, Kam BL, van Eijck CH, van Essen M, Kooij PP, Feelders RA, van Aken MO and Krenning EP: Treatment with the radiolabeled somatostatin analog [177 Lu-DOTA 0,Tyr3] octreotate: Toxicity, efficacy, and survival. J Clin Oncol 26: 2124-2130, 2008.

6. Strosberg J, El-Haddad G, Wolin E, Hendifar A, Yao J, Chasen B, Mittra E, Kunz PL, Kulke MH, Jacene H, et al: Phase 3 trial of ${ }^{177} \mathrm{Lu}$-dotatate for midgut neuroendocrine tumors. N Engl J Med 376: 125-135, 2017.

7. van der Zwan WA, Bodei L, Mueller-Brand J, de Herder WW, Kvols LK and Kwekkeboom DJ. GEPNETs update: Radionuclide therapy in neuroendocrine tumors. Eur J Endocrinol 172: R1-R8, 2015.

8. Zhang J, Kulkarni HR, Singh A, Niepsch K, Muller D and Baum RP: Peptide receptor radionuclide therapy in grade 3 neuroendocrine neoplasms: Safety and survival analysis in 69 patients. J Nucl Med 60: 377-385, 2019.

9. Mahjoub AR and O'Reilly EM: Emerging therapies for pancreas neuroendocrine cancers. Chin Clin Oncol 2: 2304-3865, 2013.

10. Strosberg JR, Fine RL, Choi J, Nasir A, Coppola D, Chen DT, Helm J and Kvols L: First-line chemotherapy with capecitabine and temozolomide in patients with metastatic pancreatic endocrine carcinomas. Cancer 117: 268-275, 2011.

11. Fine RL, Gulati AP, Krantz BA, Moss RA, Schreibman S, Tsushima DA, Mowatt KB, Dinnen RD, Mao Y, Stevens PD, et al: Capecitabine and temozolomide (CAPTEM) for metastatic, well-differentiated neuroendocrine cancers: The pancreas center at Columbia university experience. Cancer Chemother Pharmacol 71: 663-670, 2013.

12. Claringbold PG, Brayshaw PA, Price RA and Turner JH: Phase II study of radiopeptide $177 \mathrm{Lu}$-octreotate and capecitabine therapy of progressive disseminated neuroendocrine tumours. Eur J Nucl Med Mol Imaging 38: 302-311, 2011.

13. Claringbold PG, Price RA and Turner JH: Phase I-II study of radiopeptide $177 \mathrm{Lu}$-octreotate in combination with capecitabine and temozolomide in advanced low-grade neuroendocrine tumors. Cancer Biother Radiopharm 27: 561-569, 2012.

14. Claringbold PG and Turner JH: Pancreatic neuroendocrine tumor control: Durable objective response to combination 177Lu-octreotate-capecitabine-temozolomide radiopeptide chemotherapy. Neuroendocrinology 103: 432-439, 2016.

15. Bison SM, Haeck JC, Bol K, Koelewijn SJ, Groen HC, Melis M, Veenland JF, Bernsen MR and de Jong M: Optimization of combined temozolomide and peptide receptor radionuclide therapy (PRRT) in mice after multimodality molecular imaging studies. EJNMMI Res 5: 62, 2015.

16. Kesavan M, Claringbold PG and Turner JH: Hematological toxicity of combined $177 \mathrm{Lu}$-octreotate radiopeptide chemotherapy of gastroenteropancreatic neuroendocrine tumors in long-term follow-up. Neuroendocrinology 99: 108-117, 2014.

17. Thakral P, Sen I, Pant V, Gupta SK, Dureja S, Kumari J, Kumar S, Un P and Malasani V: Dosimetric analysis of patients with gastro entero pancreatic neuroendocrine tumors (NETs) treated with PRCRT (peptide receptor chemo radionuclide therapy) using Lu-177 DOTATATE and capecitabine/temozolomide (CAP/TEM). Br J Radiol 91: 20170172, 2018.

18. Stueven AK, Kayser A, Wetz C, Amthauer H, Wree A, Tacke F, Wiedenmann B, Roderburg C and Jann H: Somatostatin analogues in the treatment of neuroendocrine tumors: Past, present and future. Int J Mol Sci 20: 3049, 2019.

19. Claringbold PG and Turner JH: NeuroEndocrine tumor therapy with lutetium-177-octreotate and everolimus (NETTLE): A phase I study. Cancer Biother Radiopharm 30: 261-269, 2015.

20. Pfeifer AK, Gregersen T, Grønbæk H, Hansen CP, Müller-Brand J, Herskind Bruun K, Krogh K, Kjaer A and Knigge U: Peptide receptor radionuclide therapy with Y-DOTATOC and (177) Lu-DOTATOC in advanced neuroendocrine tumors: Results from a Danish cohort treated in Switzerland. Neuroendocrinology 93: 189-196, 2011.

21. Imhof A, Brunner P, Marincek N, Briel M, Schindler C, Rasch H, Mäcke HR, Rochlitz C, Müller-Brand J and Walter MA: Response, survival, and long-term toxicity after therapy with the radiolabeled somatostatin analogue [90Y-DOTA]-TOC in metastasized neuroendocrine cancers. J Clin Oncol 29: 2416-2423, 2011.

22. Bodei L, Cremonesi M, Grana CM, Fazio N, Iodice S, Baio SM, Bartolomei M, Lombardo D, Ferrari ME, Sansovini M, et al: Peptide receptor radionuclide therapy with (1)(7)(7) Lu-DOTATATE: The IEO phase I-II study. Eur J Nucl Med Mol Imaging 38: 2125-2135, 2011. 
23. Bodei L, Cremonesi M, Zoboli S, Grana C, Bartolomei M, Rocca P, Caracciolo M, Mäcke HR, Chinol M and Paganelli G: Receptor-mediated radionuclide therapy with 90Y-DOTATOC in association with amino acid infusion: A phase I study. Eur J Nucl Med Mol Imaging 30: 207-216, 2003.

24. Shapiro M, Wald U, Simchen E, Pomeranz S, Zagzag D, Michowiz SD, Samuel-Cahn E, Wax Y, Shuval R, Kahane Y, et al: Randomized clinical trial of intra-operative antimicrobial prophylaxis of infection after neurosurgical procedures. J Hosp Infect 8: 283-295, 1986.

25. Waldherr C, Pless M, Maecke HR, Haldemann A and Mueller-Brand J. The clinical value of [90Y-DOTA]-D-Phe1Tyr3-octreotide (90Y-DOTATOC) in the treatment of neuroendocrine tumours: A clinical phase II study. Ann Oncol 12: 941-945, 2001

26. Waldherr C, Pless M, Maecke HR, Schumacher T, Crazzolara A, Nitzsche EU, Haldemann A and Mueller-Brand J: Tumor response and clinical benefit in neuroendocrine tumors after $7.4 \mathrm{GBq}(90)$ Y-DOTATOC. J Nucl Med 43: 610-616, 2002.

27. van Essen M, Krenning EP, Kam BL, de Herder WW, van Aken MO and Kwekkeboom DJ: Report on short-term side effects of treatments with $177 \mathrm{Lu}$-octreotate in combination with capecitabine in seven patients with gastroenteropancreatic neuroendocrine tumours. Eur J Nucl Med Mol Imaging 35: 743-748, 2008.

28. Kong G, Thompson M, Collins M, Herschtal A, Hofman MS Johnston V, Eu P, Michael M and Hicks RJ: Assessment of predictors of response and long-term survival of patients with neuroendocrine tumour treated with peptide receptor chemoradionuclide therapy (PRCRT). Eur J Nucl Med Mol Imaging 41: 1831-1844, 2014

29. O'Toole D, Kianmanesh R and Caplin M: ENETS 2016 consensus guidelines for the management of patients with digestive neuroendocrine tumors: An update. Neuroendocrinology 103: 117-118, 2016.

30. Newman CB, Melmed S, Snyder PJ, Young WF, Boyajy LD Levy R, Stewart WN, Klibanski A, Molitch ME and Gagel RF: Safety and efficacy of long-term octreotide therapy of acromegaly: Results of a multicenter trial in 103 patients-a clinical research center study. J Clin Endocrinol Metab 80: 2768-2775, 1995.

31. Lamberts SW, van der Lely AJ, de Herder WW and Hofland LJ: Octreotide. N Engl J Med 334: 246-254, 1996.

32. Bodei L, Cremonesi M, Grana C, Rocca P, Bartolomei M, Chinol $\mathrm{M}$ and Paganelli G: Receptor radionuclide therapy with 90Y-[DOTA]0-Tyr3-octreotide (90Y-DOTATOC) in neuroendocrine tumours. Eur J Nucl Med Mol Imaging 31: 1038-1046, 2004
33. Bodei L, Mueller-Brand J, Baum RP, Pavel ME, Hörsch D, O'Dorisio MS, O'Dorisio TM, Howe JR, Cremonesi M, Kwekkeboom DJ and Zaknun JJ: The joint IAEA, EANM, and SNMMI practical guidance on peptide receptor radionuclide therapy (PRRNT) in neuroendocrine tumours. Eur J Nucl Med Mol Imaging 40: 800-816, 2013.

34. Bergsma H, Konijnenberg MW, van der Zwan WA, Kam BL, Teunissen JJ, Kooij PP, Mauff KAL, Krenning EP and Kwekkeboom DJ: Nephrotoxicity after PRRT with (177) Lu-DOTA-octreotate. Eur J Nucl Med Mol Imaging 43: 1802-1811, 2016.

35. Kashyap R, Jackson P, Hofman MS, Eu P, Beauregard JM, Zannino D and Hicks RJ: Rapid blood clearance and lack of long-term renal toxicity of 177Lu-DOTATATE enables shortening of renoprotective amino acid infusion. Eur J Nucl Med Mol Imaging 40: 1853-1860, 2013.

36. Kesavan M and Turner JH: Myelotoxicity of peptide receptor radionuclide therapy of neuroendocrine tumors: A decade of experience. Cancer Biother Radiopharm 31: 189-198, 2016.

37. Ploeckinger U, Kloeppel G, Wiedenmann B, Lohmann R; Representatives of 21 German NET Centers: The German NET-registry: An audit on the diagnosis and therapy of neuroendocrine tumors. Neuroendocrinology 90: 349-363, 2009.

38. Garcia-Carbonero R, Capdevila J, Crespo-Herrero G, Díaz-Pérez JA, Martínez Del Prado MP, Alonso Orduña V, Sevilla-García I, Villabona-Artero C, Beguiristain-Gómez A, Llanos-Muñoz M, et al: Incidence, patterns of care and prognostic factors for outcome of gastroenteropancreatic neuroendocrine tumors (GEP-NETs): Results from the national cancer registry of Spain (RGETNE). Ann Oncol 21: 1794-1803, 2010.

39. Lombard-Bohas C,Mitry E, O'Toole D, Louvet C,Pillon D, Cadiot G, Borson-Chazot F, Aparicio T, Ducreux M, Lecomte T, et al: Thirteen-month registration of patients with gastroenteropancreatic endocrine tumours in France. Neuroendocrinology 89: 217-222, 2009.

40. Yao JC, Hassan M, Phan A, Dagohoy C, Leary C, Mares JE, Abdalla EK, Fleming JB, Vauthey JN, Rashid A and Evans DB: One hundred years after 'carcinoid': Epidemiology of and prognostic factors for neuroendocrine tumors in 35,825 cases in the United States. J Clin Oncol 26: 3063-3072, 2008.

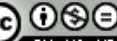

This work is licensed under a Creative Commons Attribution-NonCommercial-NoDerivatives 4.0 International (CC BY-NC-ND 4.0) License. 